\title{
$\mathrm{SC}$

OPEN Data Descriptor: Human

\section{pluripotent stem cell derived HLC transcriptome data enables molecular dissection of}

Received: 4 October 2017 Accepted: 16 January 2018

Published: 13 March 2018

\section{hepatogenesis}

\section{Wasco Wruck $^{1} \&$ James Adjaye ${ }^{1}$}

Induced pluripotent stem cells (iPSCs) and human embryonic stem cells (hESCs) differentiated into hepatocyte-like cells (HLCs) provide a defined and renewable source of cells for drug screening, toxicology and regenerative medicine. We previously reprogrammed human fetal foreskin fibroblast cells (HFF1) into iPSCs employing an episomal plasmid-based integration-free approach, this IPSC-line and the hESC lines $\mathrm{H} 1$ and $\mathrm{H} 9$ were used to model hepatogenesis in vitro. Biochemical characterisation confirmed glycogen storage, ICG uptake and release, urea and bile acid production, as well as CYP3A4 activity. Microarraybased transcriptome analyses was carried out using RNA isolated from the undifferentiated pluripotent stem cells and subsequent differentiation stages- definitive endoderm (DE) hepatic endoderm (HE) and HLCs. K-means identified 100 distinct clusters, for example, POU5F1/OCT4 marking the undifferentiated stage, SOX17 the DE stage, HNF4 $\alpha$ the HE stage, and ALB specific to HLCs, fetal liver and primary human hepatocytes $(\mathrm{PHH})$. This data descriptor describes these datasets which should be useful for gaining new insights into the molecular basis of hepatogenesis and associated gene regulatory networks.

\begin{tabular}{|c|c|}
\hline Design Type(s) & 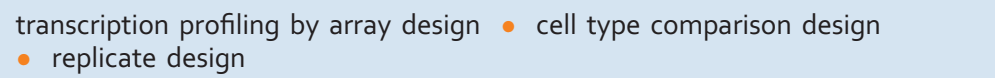 \\
\hline Measurement Type(s) & transcription profiling assay \\
\hline Technology Type(s) & DNA microarray \\
\hline Factor Type(s) & biological replicate \\
\hline Sample Characteristic(s) & 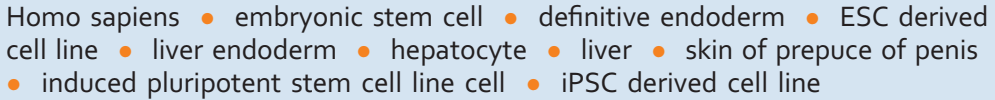 \\
\hline
\end{tabular}

${ }^{1}$ Medical Faculty, Institute for Stem Cell Research and Regenerative Medicine, Heinrich Heine University, 40225 Düsseldorf, Germany. Correspondence and requests for materials should be addressed to J. A. (email:James.Adjaye@med.uni-duesseldorf.de). 


\section{Background \& Summary}

The implementation of a well-characterized renewable source of hepatocytes differentiated from iPSCs and hESCs provides a powerful in vitro model system for analysing the molecular mechanisms associated with hepatogenesis. Several essential initiators of hepatogenesis such as fibroblast growth factor 2 and 4 (FGF4 and FGF2) $)^{1,2}$, bone morphogenic protein (BMP2), hepatocyte growth factor (HGF), oncostatin M and dexamethasone ${ }^{4}$ have already been described. These factors are sequentially supplemented into the media during the course of the differentiation process.

Besides detoxification the liver is responsible for a number of essential functions e.g. the uptake and storage of glycogen, various metabolic functions, synthesis of bile acids and production of plasma proteins. Available liver cellular models have disadvantages: (i) liver biopsy derived primary human hepatocytes $(\mathrm{PHH})$ cannot be expanded for long periods in vitro, are often obtained from diseased individuals and are difficult to obtain in sufficient quantities ${ }^{5,6}$, (ii) transformed, permanent cell lines, such as HepG2 and HepaRG, have cancer phenotypes which are significantly diverged from normal primary hepatocytes ${ }^{7-9}$. A potential alternative could be the differentiation into hepatocyte-like cells. Although hepatocyte-like cells (HLCs) derived from iPSCs are not fully mature compared to liver biopsy derived adult hepatocytes they are endowed with many advantages, for example easily generated from iPSCs, known genetic background and disease states thus optimal for disease modelling in vitro, toxicology studies and drug screening. iPSC-based cellular models have already been employed in several studies for drug screening, toxicology studies and disease modeling ${ }^{10-14}$.

The liver develops in a stepwise process in vivo: first, competence is established in the foregut endoderm in response to signals emanating from cardiac mesoderm, thereafter liver-specific gene expression is initiated ${ }^{15}$. The differentiation of hiPSCs and hESCs also proceed via the intermediate step of definitive endoderm, the bipotential hepatic endoderm, then maturation into $\mathrm{HLCs}^{16}$. Distinct stage specific changes in the associated transcriptional regulatory networks control the different phases of hepatogenesis ${ }^{17}$. Wang et al. describe a developmental progression from unmarked chromatin to poised chromatin and then to histone H3K27 acetylation which is accompanied by specific transcription factor classes $^{18}$. They suggest FOXA transcription factors - known as pioneer factors facilitating the unwinding of chromatin - to play a role at poised enhancers while lineage-specific factors such as PDX1 for pancreatic and HNF4 $\alpha$ for hepatic lineage drive the poised to an active enhancer state ${ }^{18}$.

Attaining maturation comparable to primary hepatocytes is still one of the most challenging issues associated HLC differentiation. Knowledge on HNF4 $\alpha$, as major transcription factor regulating hepatic differentiation and maturation has already been described ${ }^{15}$. Additionally Li et al. reported that HNF4 $\alpha$ lies upstream of the transcription factors HNF1 $\alpha$ and PXR suggesting it might initiate a cascade of gene regulatory networks driving hepatocyte differentiation ${ }^{15}$. In our publication related to the hESCs and hiPSC dataset pertinent to this data descriptor we confirmed expression of maturation markers such as $A L B, H N F 4 \alpha, H N F 1 \alpha$ and TTR ${ }^{16,17}$.

The data described consists of microarray gene expression data from hESCs and hiPSCs differentiated into HLCs via the DE and HE stages and also fetal liver and primary human hepatocyte samples as reference. Although transcription factors central to hepatogenesis have been described, the datasets described here will enable a more detailed analyses of gene regulatory networks associated with modelling hepatogenesis using pluripotent stem cells.

\section{Methods}

\section{Human ES and iPS cells culture}

Human ES cell lines H1 and H9 (WiCell Research Institute, Madison, Wisconsin) from passage 39 to 66 were maintained under sterile conditions in a humidified incubator in a 5\% CO2-95\% air atmosphere at $37^{\circ} \mathrm{C}$ (INNOVA CO-170 Incubator, New Brunswick Scientific). In a routine culture cells were maintained on Matrigel $^{\bullet}$ in conditioned media $(\mathrm{CM})^{19}$. Under these culture conditions, hESCs were confirmed to stain positive for OCT4, SSEA-4, TRA-1-60, and TRA-1-81 (ES Cell Characterization Kit, Chemicon). Before initiating the differentiation cells were washed with PBS without $\mathrm{Ca} 2+\mathrm{Mg} 2+$ (Gibco, Invitrogen).

Cell culture of iPS cells which were derived from Human neonatal foreskin fibroblasts HFF1 is described in Matz et al. ${ }^{17}$.

\section{Differentiation into hepatocyte-like cells (HLCs)}

The derivation of HLCs from the hESC lines $\mathrm{H} 1$ and $\mathrm{H} 9^{16}$ followed protocols described by Hay et al. ${ }^{20}$ and Agarwal et al. ${ }^{21}$. RNA samples were extracted after each step of the differentiation protocol.

Differentiation of iPS cells into $\mathrm{HLCs}^{17}$ followed in large parts the protocol described by Jozefczuk et al. ${ }^{16}$.

The overall experimental design of this study is illustrated in Figure 1a. Two pluripotent stem cells lines (hESC- H1 and H9) and fetal foreskin derived iPSC were used. Both proceeded via the intermediate DE and HE stages to HLCs and then compared to commercially bought RNA from fetal liver (Stratagene, MVP Total RNA: tissue from single male donor, 18th week of gestation; positive control for the iPSCbased differentiations: Clontech, \#636540) and adult liver biopsy-derived primary human hepatocytesPHH (Ready Heps Fresh Hepatocytes; Lonza, 65-year old male of Asian origin; positive control for the iPSC-based differentiations: Clontech, \#636531). 
a

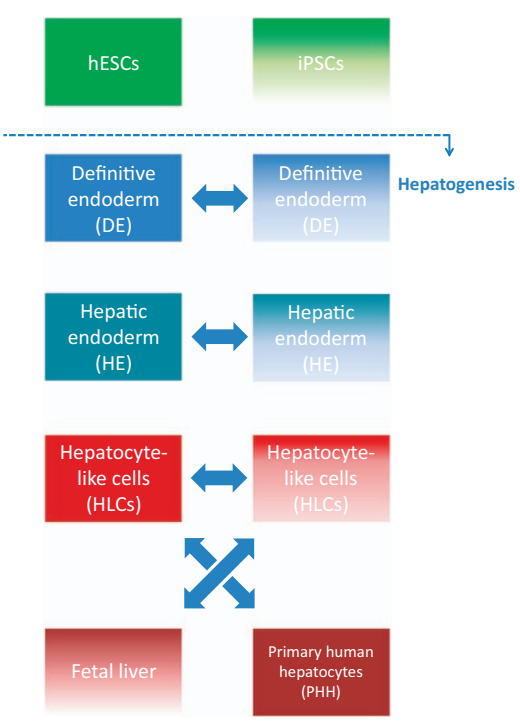

b

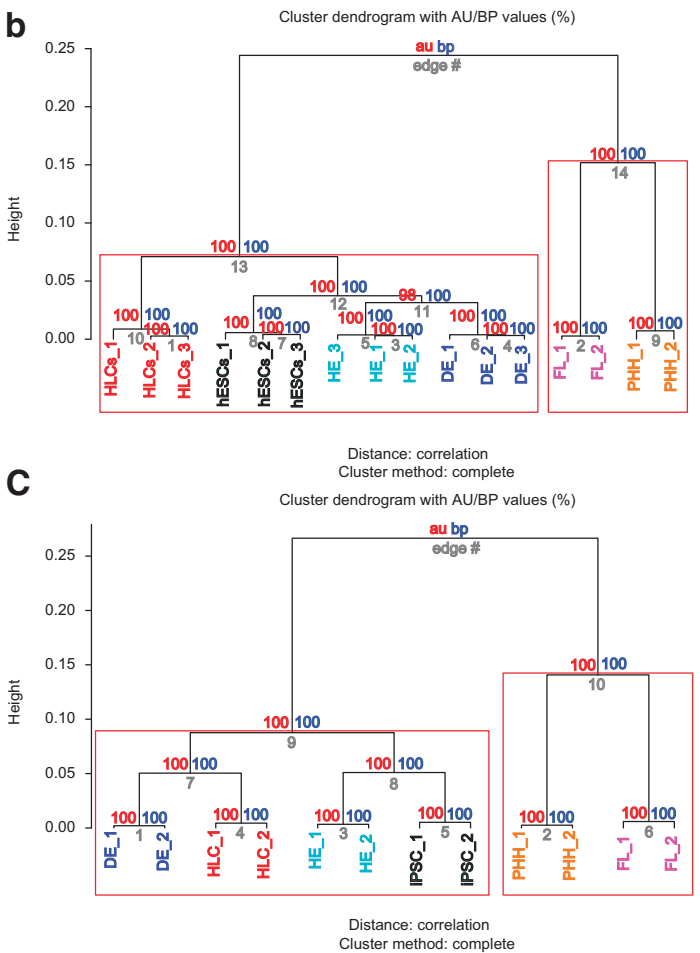

Figure 1. Comparison of hepatic differentiation of iPSCs and hESCs. (a) Scheme of comparative hepatic differentiation of iPSCs and hESCs. iPSCs and hESCs are differentiated into HLCs which can be compared versus each other and versus fetal liver (FL) and PHHs. Also the intermediate stages DE and HE are captured and thus can be subjected to comparative analysis. Hierarchical clustering of hESC (b) and iPSC (c) differentiation into HLCs was performed via pvclust using 1000 bootstrap samples. „au“ (approximately unbiased) is computed by multiscale bootstrap resampling and „bp“ (bootstrap probability) by normal bootstrapping. Red rectangles mark clusters with AU larger than 95\%. Thus the dendrogram is with one exception in hESC (98\%) at 100\% supported by data. All replicates cluster together. HLCs cluster apart from hESCs/iPSCs, DE and HE. Fetal liver and PHH cluster together and separated from the hESC/iPSC-derived hepatic differentiation stages.

\section{Illumina BeadChip hybridisation}

Biotin-labelled cRNA was produced by means of a linear amplification kit (Ambion, Austin, TX, USA) using $500 \mathrm{ng}$ of quality-checked total RNA as input. Chip hybridisations, washing, Cy3-streptavidin staining, and scanning were performed on an Illumina BeadStation 500 platform (Il-lumina, San Diego, CA, USA) using reagents and following protocols supplied by the manufacturer. cRNA samples were hybridised in biological triplicates on HumanRef- 8 Expression BeadChips. The following samples were hybridized: Undifferentiated cells (H1 cell line), DE (definitive endoderm)-differentiated cells, HE (hepatic endoderm)-differentiated cells and hepatocyte-like cells (HLCs) derived with two independent protocols $^{20,21}$.

Differentiation experiments of iPSCs were hybridised on Illumina HumanHT-12 BeadChips. For details see the Methods description in Matz et al. ${ }^{17}$.

The summary of bead-level data to bead-summary data was carried out using the manufacturer's software BeadStudio 3.0 (Illumina) for hESC and iPSC differentiation experiments. Table 1 provides an overview of all samples used for this study.

\section{Data analysis and statistical methods}

For further analysis, the bead-summary data saved in the BeadStudio was imported into the Bioconductor environment $^{22}$ and quantile normalized using the bioconductor package lumi ${ }^{23}$. Global gene expression similarities within biological replicates and between dedicated differentiation stages, pairwise Pearson correlation coefficients were calculated for all samples. Cluster analyses were performed using the R/ Bioconductor environment ${ }^{22}$ and the package pvclust ${ }^{24}$ using $\mathrm{n}=1000$ for bootstrap sampling. $\mathrm{k}$-means clustering was employed to identify clusters of genes with similar gene expression changes over the stages of the differentiation protocol using $\mathrm{k}=100$ as number of clusters. The software is available in the Supplementary Data File 1. 


\begin{tabular}{|c|c|c|c|c|}
\hline ID & description & replicate $[\#]$ & NCBI GEO sample & NCBI GEO accession no. \\
\hline hESCs_1 & human embryonic stem cells (H1) & 1 & GSM2683216 & GSE100447 \\
\hline hESCs_2 & human embryonic stem cells (H1) & 2 & GSM2683217 & GSE100447 \\
\hline hESCs_3 & human embryonic stem cells (H1) & 3 & GSM2683218 & GSE100447 \\
\hline DE_hESCs_1 & definite endoderm from $\mathrm{hESCs}$ & 1 & GSM2683219 & GSE100447 \\
\hline DE_hESCs_2 & definite endoderm from $\mathrm{hESCs}$ & 2 & GSM2683220 & GSE100447 \\
\hline DE_hESCs_3 & definite endoderm from hESCs & 3 & GSM2683221 & GSE100447 \\
\hline HE_hESCs_1 & hepatic endoderm from hESCs & 1 & GSM2683222 & GSE100447 \\
\hline HE_hESCs_2 & hepatic endoderm from hESCs & 2 & GSM2683223 & GSE100447 \\
\hline HE_hESCs_3 & hepatic endoderm from hESCs & 3 & GSM2683224 & GSE100447 \\
\hline HLCs_hESCs_1 & hepatocyte-like cells from hESCs & 1 & GSM2683225 & GSE100447 \\
\hline HLCs_hESCs_2 & hepatocyte-like cells from hESCs & 2 & GSM2683226 & GSE100447 \\
\hline HLCs_hESCs_3 & hepatocyte-like cells from hESCs & 3 & GSM2683227 & GSE100447 \\
\hline Fetal_Liver_1 & fetal liver & 1 & GSM2683228 & GSE100447 \\
\hline Fetal_Liver_2 & fetal liver & 2 & GSM2683229 & GSE100447 \\
\hline PHH_1 & primary human hepatocytes & 1 & GSM2683230 & GSE100447 \\
\hline PHH_2 & primary human hepatocytes & 2 & GSM2683231 & GSE100447 \\
\hline iPSCs_1 & induced pluripotent stem cells & 1 & GSM1618658 & GSE66282 \\
\hline iPSCs_2 & induced pluripotent stem cells & 2 & GSM1618659 & GSE66282 \\
\hline DE_iPSCs_1 & definite endoderm from iPSCs & 1 & GSM1618660 & GSE66282 \\
\hline DE_iPSCs_2 & definite endoderm from iPSCs & 2 & GSM1618661 & GSE66282 \\
\hline HE_iPSCs_1 & hepatic endoderm from iPSCs & 1 & GSM1618662 & GSE66282 \\
\hline HE_iPSCs_2 & hepatic endoderm from iPSCs & 2 & GSM1618663 & GSE66282 \\
\hline HLCs_iPSCs_1 & hepatocyte-like cells from iPSCs & 1 & GSM1618664 & GSE66282 \\
\hline HLCs_iPSCs_2 & hepatocyte-like cells from iPSCs & 2 & GSM1618665 & GSE66282 \\
\hline fetal_liver_1 & fetal liver & 1 & GSM1618666 & GSE66282 \\
\hline fetal_liver_2 & fetal liver & 2 & GSM1618667 & GSE66282 \\
\hline PHH_1 & primary human hepatocytes & 1 & GSM1618668 & GSE66282 \\
\hline PHH_2 & primary human hepatocytes & 2 & GSM1618669 & GSE66282 \\
\hline
\end{tabular}

Table 1. Samples related to data sets in repositories.

\section{Data Records}

\section{Data Record 1}

The iPSC-related microarray experiments related to this publication have been performed on the Illumina BeadStation 500 platform (Illumina, San Diego, CA, USA) using the Illumina HumanHT-12 BeadChip. The data were uploaded to NCBI GEO and are accessible under (Data Citation 1). The dataset (Data Citation 1) was first released to the public with the publication Matz et al. ${ }^{17}$.

\section{Data Record 2}

The hESC-related microarray experiments related to this publication have been performed on the Illumina BeadStation 500 platform (Illumina, San Diego, CA, USA) using the HumanRef-8 Expression BeadChip. The data were uploaded to NCBI GEO and are accessible under (Data Citation 2). The dataset (Data Citation 2) is being released for the first time with the publication of this Data Descriptor.

\section{Technical Validation}

\section{Transcriptome data}

Microarray data were quality controlled via the proprietary Illumina quality control mechanisms. Tables of Pearson correlation coefficients of all samples vs. each other were generated validating the absence of outliers (Tables 2 and 3). Several samples were investigated in triplicates, all others in duplicates. Figure $1 \mathrm{~b}$ and Figure $1 \mathrm{c}$ show that the replicates cluster together as well in the hESC as in the iPSC differentiation experiments as one would expect. Both bootstrap sampling methods implemented in the pvclust clustering software confirmed that all clusters within the dendrogram are with one exception (98\%) at $100 \%$ supported by data. This demonstrates the validity of experiments on the level of wholegenome gene expression. 


\begin{tabular}{|c|c|c|c|c|c|c|c|c|c|c|c|c|c|c|c|c|}
\hline sample & hESCs_1 & hESCs_2 & hESCs_3 & DE_1 & DE_2 & DE_3 & HE_1 & HE_2 & HE_3 & HLCs_1 & HLCs_2 & HLCs_3 & Fetal_Liver_1 & Fetal_Liver_2 & PHH_1 & PHH_2 \\
\hline hESCs_1 & 1.0000 & 0.9927 & 0.9913 & 0.9489 & 0.9455 & 0.9463 & 0.9534 & 0.9539 & 0.9564 & 0.9117 & 0.9057 & 0.9081 & 0.6798 & 0.6787 & 0.6062 & 0.6079 \\
\hline hESCs_2 & 0.9927 & 1.0000 & 0.9964 & 0.9483 & 0.9458 & 0.9463 & 0.9530 & 0.9538 & 0.9567 & 0.9094 & 0.9081 & 0.9100 & 0.6910 & 0.6919 & 0.6103 & 0.6113 \\
\hline hESCs_3 & 0.9913 & 0.9964 & 1.0000 & 0.9477 & 0.9463 & 0.9460 & 0.9539 & 0.9544 & 0.9563 & 0.9177 & 0.9182 & 0.9206 & 0.6903 & 0.6907 & 0.6114 & 0.6124 \\
\hline DE_1 & 0.9489 & 0.9483 & 0.9477 & 1.0000 & 0.9963 & 0.9968 & 0.9631 & 0.9631 & 0.9640 & 0.9308 & 0.9270 & 0.9273 & 0.6743 & 0.6725 & 0.6133 & 0.6149 \\
\hline DE_2 & 0.9455 & 0.9458 & 0.9463 & 0.9963 & 1.0000 & 0.9978 & 0.9620 & 0.9613 & 0.9618 & 0.9255 & 0.9255 & 0.9281 & 0.6663 & 0.6645 & 0.6097 & 0.6115 \\
\hline DE_3 & 0.9463 & 0.9463 & 0.9460 & 0.9968 & 0.9978 & 1.0000 & 0.9597 & 0.9586 & 0.9601 & 0.9213 & 0.9200 & 0.9220 & 0.6662 & 0.6643 & 0.6113 & 0.6135 \\
\hline HE_1 & 0.9534 & 0.9530 & 0.9539 & 0.9631 & 0.9620 & 0.9597 & 1.0000 & 0.9981 & 0.9974 & 0.9453 & 0.9422 & 0.9447 & 0.6805 & 0.6799 & 0.6224 & 0.6234 \\
\hline HE_2 & 0.9539 & 0.9538 & 0.9544 & 0.9631 & 0.9613 & 0.9586 & 0.9981 & 1.0000 & 0.9975 & 0.9458 & 0.9432 & 0.9451 & 0.6831 & 0.6825 & 0.6211 & 0.6223 \\
\hline HE_3 & 0.9564 & 0.9567 & 0.9563 & 0.9640 & 0.9618 & 0.9601 & 0.9974 & 0.9975 & 1.0000 & 0.9411 & 0.9379 & 0.9398 & 0.6812 & 0.6809 & 0.6211 & 0.6226 \\
\hline HLCs_1 & 0.9117 & 0.9094 & 0.9177 & 0.9308 & 0.9255 & 0.9213 & 0.9453 & 0.9458 & 0.9411 & 1.0000 & 0.9871 & 0.9834 & 0.6912 & 0.6889 & 0.6063 & 0.6086 \\
\hline HLCs_2 & 0.9057 & 0.9081 & 0.9182 & 0.9270 & 0.9255 & 0.9200 & 0.9422 & 0.9432 & 0.9379 & 0.9871 & 1.0000 & 0.9972 & 0.6931 & 0.6923 & 0.6121 & 0.6149 \\
\hline HLCs_3 & 0.9081 & 0.9100 & 0.9206 & 0.9273 & 0.9281 & 0.9220 & 0.9447 & 0.9451 & 0.9398 & 0.9834 & 0.9972 & 1.0000 & 0.6854 & 0.6849 & 0.6110 & 0.6139 \\
\hline Fetal_Liver_1 & 0.6798 & 0.6910 & 0.6903 & 0.6743 & 0.6663 & 0.6662 & 0.6805 & 0.6831 & 0.6812 & 0.6912 & 0.6931 & 0.6854 & 1.0000 & 0.9979 & 0.7384 & 0.7364 \\
\hline Fetal_Liver_2 & 0.6787 & 0.6919 & 0.6907 & 0.6725 & 0.6645 & 0.6643 & 0.6799 & 0.6825 & 0.6809 & 0.6889 & 0.6923 & 0.6849 & 0.9979 & 1.0000 & 0.7422 & 0.7403 \\
\hline PHH_1 & 0.6062 & 0.6103 & 0.6114 & 0.6133 & 0.6097 & 0.6113 & 0.6224 & 0.6211 & 0.6211 & 0.6063 & 0.6121 & 0.6110 & 0.7384 & 0.7422 & 1.0000 & 0.9973 \\
\hline PHH_2 & 0.6079 & 0.6113 & 0.6124 & 0.6149 & 0.6115 & 0.6135 & 0.6234 & 0.6223 & 0.6226 & 0.6086 & 0.6149 & 0.6139 & 0.7364 & 0.7403 & 0.9973 & 1.0000 \\
\hline
\end{tabular}

Table 2. Pearson correlation coefficients of hESC-derived transcriptome data of all samples vs. each other.

\begin{tabular}{|l|l|l|l|l|l|l|l|l|l|l|l|l|}
\hline sample & iPSC_B1_1 & iPSC_B1_2 & DE_1 & DE_2 & HE_1 & HE_2 & HLC_1 & HLC_2 & fetal_liver_1 & fetal_liver_2 & PHH_1 & PHH_2 \\
\hline iPSC_B1_1 & 1.0000 & 0.9948 & 0.9356 & 0.9387 & 0.9502 & 0.9490 & 0.9155 & 0.9214 & 0.8150 & 0.8244 & 0.7413 & 0.7336 \\
\hline iPSC_B1_2 & 0.9948 & 1.0000 & 0.9419 & 0.9448 & 0.9541 & 0.9527 & 0.9228 & 0.9274 & 0.8236 & 0.8331 & 0.7512 & 0.7431 \\
\hline DE_1 & 0.9356 & 0.9419 & 1.0000 & 0.9980 & 0.9202 & 0.9124 & 0.9546 & 0.9496 & 0.8191 & 0.8285 & 0.7444 & 0.7363 \\
\hline DE_2 & 0.9387 & 0.9448 & 0.9980 & 1.0000 & 0.9238 & 0.9165 & 0.9548 & 0.9508 & 0.8199 & 0.8297 & 0.7453 & 0.7371 \\
\hline HE_1 & 0.9502 & 0.9541 & 0.9202 & 0.9238 & 1.0000 & 0.9966 & 0.9225 & 0.9363 & 0.8360 & 0.8379 & 0.7643 & 0.7581 \\
\hline HE_2 & 0.9490 & 0.9527 & 0.9124 & 0.9165 & 0.9966 & 1.0000 & 0.9165 & 0.9311 & 0.8328 & 0.8345 & 0.7639 & 0.7582 \\
\hline HLC_1 & 0.9155 & 0.9228 & 0.9546 & 0.9548 & 0.9225 & 0.9165 & 1.0000 & 0.9956 & 0.8399 & 0.8488 & 0.7714 & 0.7642 \\
\hline HLC_2 & 0.9214 & 0.9274 & 0.9496 & 0.9508 & 0.9363 & 0.9311 & 0.9956 & 1.0000 & 0.8440 & 0.8510 & 0.7753 & 0.7685 \\
\hline fetal_liver_1 & 0.8150 & 0.8236 & 0.8191 & 0.8199 & 0.8360 & 0.8328 & 0.8399 & 0.8440 & 1.0000 & 0.9941 & 0.8684 & 0.8624 \\
\hline fetal_liver_2 & 0.8244 & 0.8331 & 0.8285 & 0.8297 & 0.8379 & 0.8345 & 0.8488 & 0.8510 & 0.9941 & 1.0000 & 0.8662 & 0.8593 \\
\hline PHH_1 & 0.7413 & 0.7512 & 0.7444 & 0.7453 & 0.7643 & 0.7639 & 0.7714 & 0.7753 & 0.8684 & 0.8662 & 1.0000 & 0.9974 \\
\hline PHH_2 & 0.7336 & 0.7431 & 0.7363 & 0.7371 & 0.7581 & 0.7582 & 0.7642 & 0.7685 & 0.8624 & 0.8593 & 0.9974 & 1.0000 \\
\hline
\end{tabular}

Table 3. Pearson correlation coefficients of iPSC-derived transcriptome data of all samples vs. each other.

\section{k-means clustering to assess differentiation stages and similarity to primary hepatocytes}

Normalized gene expression microarray data of the iPSC differentiation experiments were further investigated via a k-means clustering algorithm. The algorithm split the data into 100 clusters of genes with similar expression over all differentiation stages. Associations of genes with clusters are included in the publication by Matz et al. ${ }^{17}$. Several clusters were representative for distinct differentiation stages. Genes from cluster\#9 were employed to make a tissue type prediction via the tool KeyGenes ${ }^{25}$ (Figure 2a). Based on the normalized gene expression data of these genes KeyGenes predicted the tissue type "liver" for HLC, fetal liver and PHH samples. Figure $2 \mathrm{~b}$ demonstrates that genes from cluster\#9 have most abundantly peaks at the HLC stages.

Furthermore, k-means clustering provided several stage-specific clusters six of which are shown in Figure 3. These represent stages iPSCs, definite endoderm, hepatic endoderm, HLCs, fetal liver and PHHs and display a gene expression peak at the dedicated stage. They include stage-specific markers which in some cases are already known: POU5F1/ OCT4 in the iPSC-cluster, SOX17 in the DE-cluster, AFP in the fetal-liver-cluster and $A L B$ in the PHH-cluster. In Supplementary Fig. S4F of our previous publication related to the iPSC datase ${ }^{17}$ of this data descriptor we could confirm PHH-cluster activity of the transcription factors HNF4 $\alpha$ and HNF1 $\alpha$ reported by $\mathrm{Li}$ et al. ${ }^{15}$ as factors inducing hepatocyte 

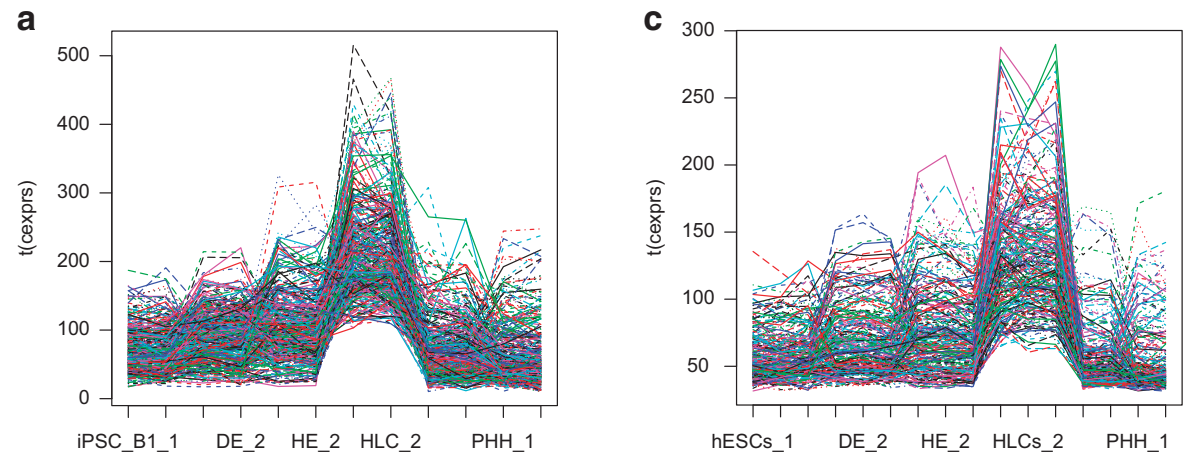

b

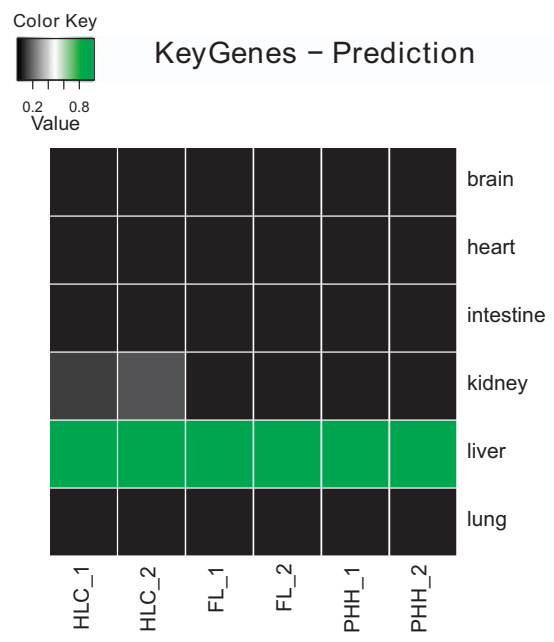

d

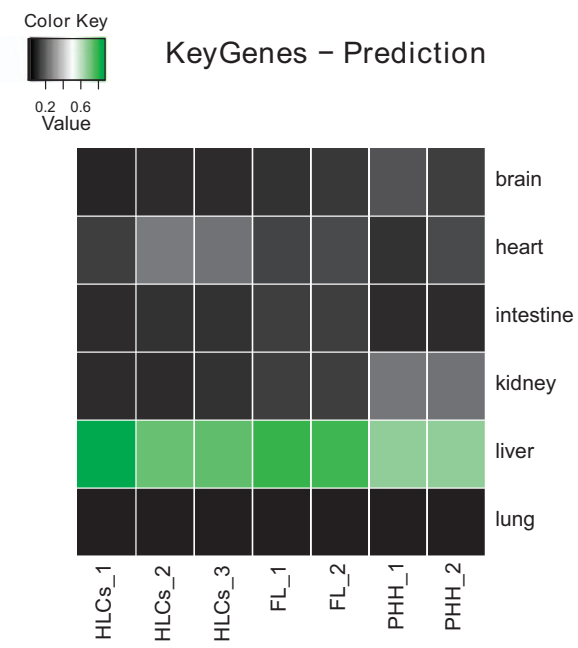

Figure 2. Characterization of hepatocyte-like cells. (a) Plot of 407 genes from the k-means cluster\#9 over all differentiation stages derived from the iPSCs. The plot demonstrates that this cluster is representative for HLCs. (b) KeyGenes tissue classification of for iPSCs k-means Hepatocyte-like-cell (HLC) cluster9 (source: Matz et $a l .{ }^{17}$ ). Based on NCBI GEO datasets for human liver, brain, intestine, kidney, lung and heart via the KeyGenes tool a training set for these Illumina microarray platform data was generated. Genes from the HLC cluster\#9 resulting from k-means clustering and HLC, fetal liver (FL) and primary human hepatocyte samples were used as test set. (c) Plot of 263 genes from the k-means cluster\#2 over all differentiation stages derived from the hESCs. The plot demonstrates that this cluster is representative for HLCs. (d) KeyGenes tissue-based classification for hESCs k-means Hepatocyte-like-cell (HLC) cluster\#2.

differentiation and furthermore reveal the activity of CTCF, ZFX, FOXA2, FOXA1, CEBPA. Additionally, these datasets may provide new insights into the differences and similarities of the hepatocyte differentiation processes between hESCs and iPSCs. Figure 4 using marker genes from the representative k-means-clusters shows that the DE stage and HLCs are very similar between hESC- and iPSC-derived differentiations while the HE stage provides a pronounced peak in iPSC-derived cells and a small peak in hESC-derived cells. As a cautionary note, the iPSC and hESC differentiations into HLCs and also the microarray-based transcriptome analyses were not conducted simultaneously, hence the observed minor variations.

\section{Usage Notes}

The microarray experiments related to this publication have been performed on the Illumina BeadStation 500 platform (Illumina, San Diego, CA, USA) but on different BeadChips. The iPSC-derived differentiations were hybridized using the Illumina HumanHT-12 BeadChip while the hESC-derived differentiations were hybridized using the HumanRef-8 Expression BeadChip. The differing chip types should be taken into account when comparing transcriptomics data between hESC-derived and iPSCderived experiments. Further points which should be considered are: (1) The fetal liver RNA was derived from liver homogenates containing all cells, but the adult liver samples were derived from isolated hepatocytes; (2) the liver disease for which the biopsy was performed could have an influence on the dataset; (3) the two different differentiation protocols used may affect the data. 
a
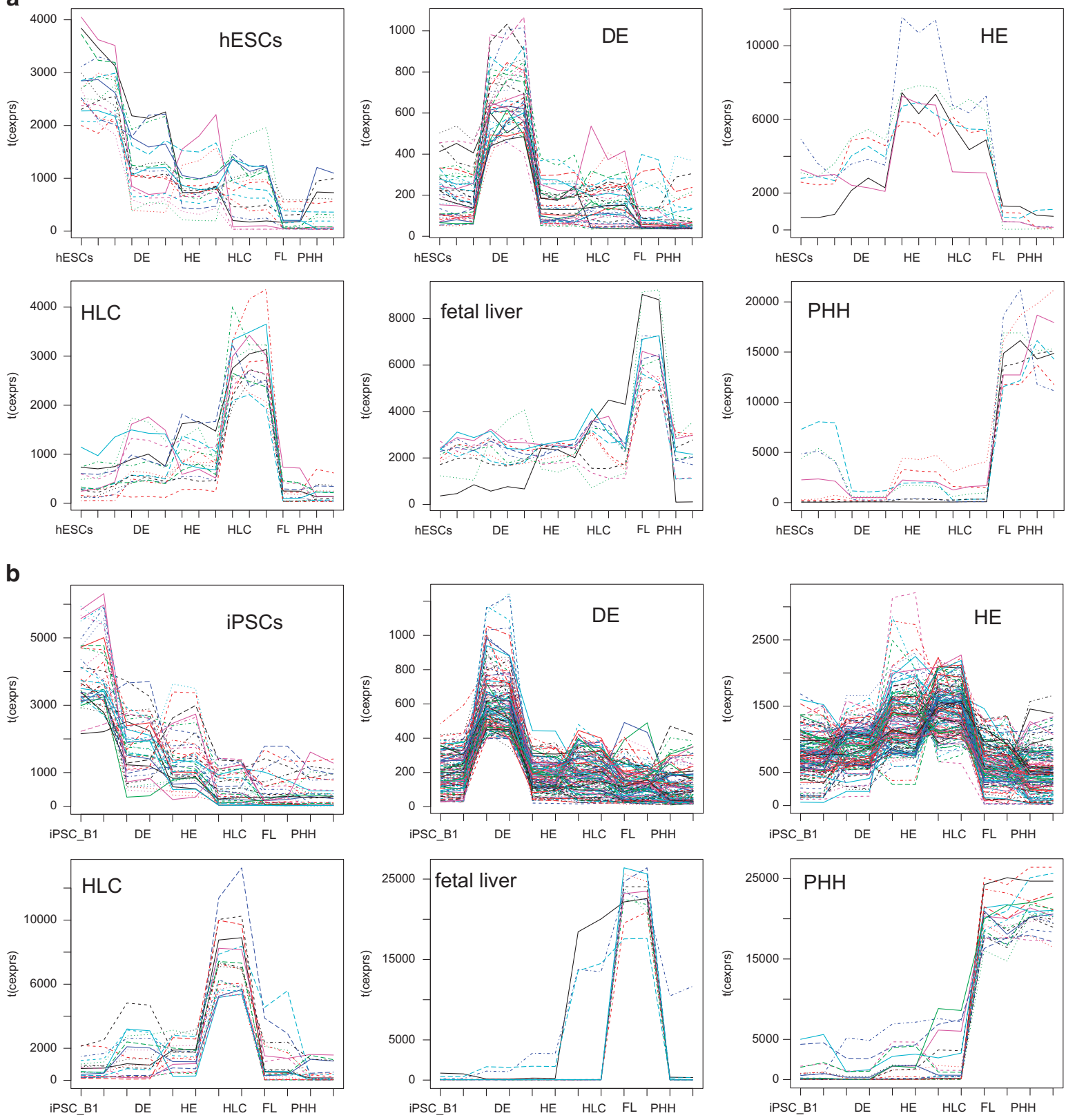

Figure 3. Clusters representative for all stages of differentiation. Via k-means clustering genes were assigned to 100 clusters having similar behaviour during the hepatic differentiation process. The plots in (a) show expression of genes in the hESC-differentiation experiments associated with cluster\#94 representative for hESCs, cluster\#23 containing SOX17 representative for DE, cluster\#64 representative for HE, cluster\#77 representative for HLCs, cluster\#19 containing AFP representative for fetal liver, cluster\#59 containing the liver marker ALB representative for PHHs. The plots in (b) show expression of genes in the iPSC-differentiation experiments associated with cluster\#68 representative for iPSCs, cluster\#81 containing SOX17 representative for DE, cluster\#37 representative for HE, cluster\#51 representative for HLCs, cluster\#72 containing AFP representative for fetal liver, cluster\#91 containing the liver marker $A L B$ representative for PHHs. 
a
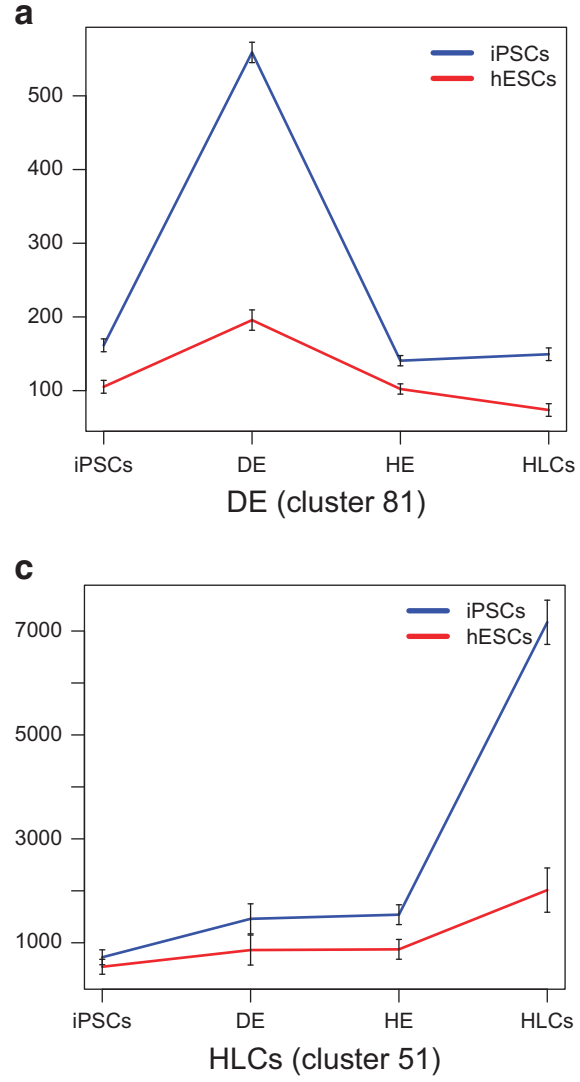

b

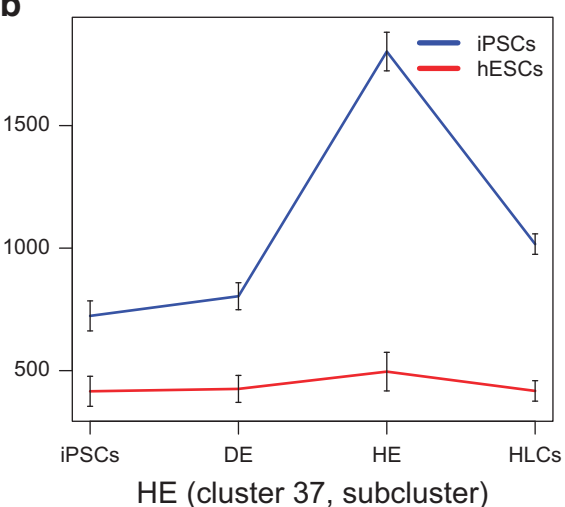

d

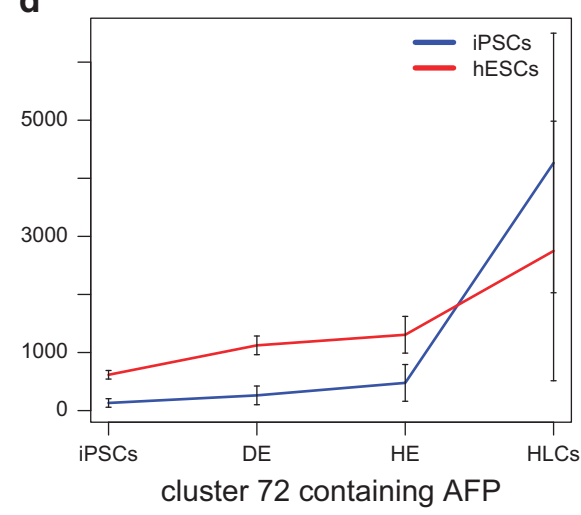

Figure 4. Comparison of clusters representative for DE, HE and HLCs between hESC- and iPSC

differentiation. Genes from representative clusters for (a) DE (cluster\#81), (b) HE (cluster\#37,subcluster), (c) HLCs (cluster\#51) and (d) cluster \#72 containing AFP were compared between hESC- and iPSC-derived lines. Mean and standard error of all genes in the dedicated clusters are shown. The DE, AFP and HLC clusters show maxima at the associated stages in both differentiations. The HE cluster provides a pronounced peak in iPSCderived cells and a small peak in hESC-derived cells.

\section{References}

1. Jung, J., Zheng, M., Goldfarb, M. \& Zaret, K. S. Initiation of mammalian liver development from endoderm by fibroblast growth factors. Science 284, 1998-2003 (1999).

2. Schwartz, R. E. et al. Defined conditions for development of functional hepatic cells from human embryonic stem cells. Stem Cells Dev. 14, 643-655 (2005).

3. Rossi, J. M., Dunn, N. R., Hogan, B. L. \& Zaret, K. S. Distinct mesodermal signals, including BMPs from the septum transversum mesenchyme, are required in combination for hepatogenesis from the endoderm. Genes Dev. 15, 1998-2009 (2001).

4. Kamiya, A., Kinoshita, T. \& Miyajima, A. Oncostatin M and hepatocyte growth factor induce hepatic maturation via distinct signaling pathways. FEBS Lett. 492, 90-94 (2001).

5. Elaut, G. et al. Molecular mechanisms underlying the dedifferentiation process of isolated hepatocytes and their cultures. Curr. Drug Metab. 7, 629-660 (2006).

6. Fraczek, J., Bolleyn, J., Vanhaecke, T., Rogiers, V. \& Vinken, M. Primary hepatocyte cultures for pharmaco-toxicological studies: at the busy crossroad of various anti-dedifferentiation strategies. Arch. Toxicol. 87, 577-610 (2013).

7. Hart, S. N. et al. A comparison of whole genome gene expression profiles of HepaRG cells and HepG2 cells to primary human hepatocytes and human liver tissues. Drug Metab. Dispos. Biol. Fate Chem 38, 988-994 (2010).

8. Gerets, H. H. J. et al. Characterization of primary human hepatocytes, HepG2 cells, and HepaRG cells at the mRNA level and CYP activity in response to inducers and their predictivity for the detection of human hepatotoxins. Cell Biol. Toxicol. 28, 69-87 (2012).

9. Jetten, M. J. A., Kleinjans, J. C. S., Claessen, S. M., Chesné, C. \& van Delft, J. H. M. Baseline and genotoxic compound induced gene expression profiles in HepG2 and HepaRG compared to primary human hepatocytes. Toxicol. Vitro Int. J. Publ. Assoc. BIBRA 27, 2031-2040 (2013).

10. Rashid, S. T. et al. Modeling inherited metabolic disorders of the liver using human induced pluripotent stem cells. J. Clin. Invest. 120, 3127-3136 (2010).

11. Sullivan, G. J. et al. Generation of functional human hepatic endoderm from human induced pluripotent stem cells. Hepatol. Baltim. Md 51, 329-335 (2010).

12. Yusa, K. et al. Targeted gene correction of $\alpha 1$-antitrypsin deficiency in induced pluripotent stem cells. Nature 478, 391-394 (2011).

13. Wruck, W., Graffmann, N., Kawala, M.-A. \& Adjaye, J. Concise Review: Current Status and Future Directions on Research Related to Nonalcoholic Fatty Liver Disease. Stem Cells Dayt. Ohio 35, 89-96 (2017). 
14. Graffmann, N. et al. Modeling Nonalcoholic Fatty Liver Disease with Human Pluripotent Stem Cell-Derived Immature Hepatocyte-Like Cells Reveals Activation of PLIN2 and Confirms Regulatory Functions of Peroxisome Proliferator-Activated Receptor Alpha. Stem Cells Dev. 25, 1119-1133 (2016).

15. Li, J., Ning, G. \& Duncan, S. A. Mammalian hepatocyte differentiation requires the transcription factor HNF-4alpha. Genes Dev. 14, 464-474 (2000).

16. Jozefczuk, J., Prigione, A., Chavez, L. \& Adjaye, J. Comparative Analysis of Human Embryonic Stem Cell and Induced Pluripotent Stem Cell-Derived Hepatocyte-Like Cells Reveals Current Drawbacks and Possible Strategies for Improved Differentiation. Stem Cells Dev. 20, 1259-1275 (2010).

17. Matz, P. et al. Footprint-free human fetal foreskin derived iPSCs: A tool for modeling hepatogenesis associated gene regulatory networks. Sci. Rep. 7, 6294 (2017).

18. Wang, A. et al. Epigenetic Priming of Enhancers Predicts Developmental Competence of hESC-Derived Endodermal Lineage Intermediates. Cell Stem Cell 16, 386-399 (2015).

19. Jozefczuk, J., Drews, K. \& Adjaye, J. Preparation of mouse embryonic fibroblast cells suitable for culturing human embryonic and induced pluripotent stem cells. J. Vis. Exp. JoVE, doi:10.3791/3854 (2012).

20. Hay, D. C. et al. Efficient differentiation of hepatocytes from human embryonic stem cells exhibiting markers recapitulating liver development in vivo. Stem Cells Dayt. Ohio 26, 894-902 (2008).

21. Agarwal, S., Holton, K. L. \& Lanza, R. Efficient differentiation of functional hepatocytes from human embryonic stem cells. Stem Cells Dayt. Ohio 26, 1117-1127 (2008).

22. Gentleman, R. C et al. Bioconductor: open software development for computational biology and bioinformatics. Genome Biol. 5, R80 (2004).

23. Du, P., Kibbe, W. A. \& Lin, S. M. lumi: a pipeline for processing Illumina microarray. Bioinformatics 24, 1547-1548 (2008).

24. Suzuki, R. \& Shimodaira, H. Pvclust: an R package for assessing the uncertainty in hierarchical clustering. Bioinformatics 22, 1540-1542 (2006).

25. Roost, M. S. et al. KeyGenes, a Tool to Probe Tissue Differentiation Using a Human Fetal Transcriptional Atlas. Stem Cell Rep 4, $1112-1124$ (2015).

\section{Data Citations}

1. NCBI Gene Expression Omnibus GSE66282 (2017).

2. NCBI Gene Expression Omnibus GSE100447 (2017).

\section{Acknowledgements}

J.A. acknowledges support from the Medical faculty of Heinrich Heine University, Düsseldorf, Germany.

\section{Author Contributions}

W.W. performed bioinformatic analyses. W.W. and J.A. wrote the manuscript. J.A. conceived the concept and coordinated the work.

\section{Additional Information}

Competing interests: The authors declare no competing financial interests.

How to cite this article: Wruck, W. \& Adjaye, J. Human pluripotent stem cell derived HLC transcriptome data enables molecular dissection of hepatogenesis. Sci. Data 5:180035 doi:10.1038/ sdata.2018.35 (2018).

Publisher's note: Springer Nature remains neutral with regard to jurisdictional claims in published maps and institutional affiliations.

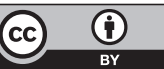

Open Access This article is licensed under a Creative Commons Attribution 4.0 International License, which permits use, sharing, adaptation, distribution and reproduction in any medium or format, as long as you give appropriate credit to the original author(s) and the source, provide a link to the Creative Commons license, and indicate if changes were made. The images or other third party material in this article are included in the article's Creative Commons license, unless indicated otherwise in a credit line to the material. If material is not included in the article's Creative Commons license and your intended use is not permitted by statutory regulation or exceeds the permitted use, you will need to obtain permission directly from the copyright holder. To view a copy of this license, visit http://creativecommons. org/licenses/by/4.0/

The Creative Commons Public Domain Dedication waiver http://creativecommons.org/publicdomain/ zero/1.0/ applies to the metadata files made available in this article.

(c) The Author(s) 2018 